\title{
A Retrospective Analysis of Prescribing Practices through WHO Prescribing Indicators at Four Selected Hospitals of West Ethiopia Jimma Likisa Lenjisa ${ }^{1 *}$ and Tadese Haile Fereja ${ }^{2}$
}

${ }^{1}$ Department of Pharmacy, College Of Medicine and Health Sciences, Clinical Pharmacy Unit, Ambo University, Ethiopia ${ }^{2}$ Department of Pharmacy, Pharmaceutical Analysis, College Of Medicine and Health Sciences, Ambo University, Ethiopia

\begin{abstract}
Background: Periodic assessment of the prescribing practices in a health facility is necessary to identify specific drug use problems, sensitize practitioners on rational drug prescription and provide policy makers with relevant information. The purpose of this survey is, therefore, to analysis the prescribing practice of clinicians using world health organization (WHO) prescribing indicators at four selected public hospitals found in west Ethiopia with ultimate goal of ensuring rational drug use.

Methods: This study was a descriptive cross-sectional survey which investigated the prescribing practices of prescribers using WHO core prescribing indicators at four selected hospitals in west Ethiopia. We have retrospectively reviewed 2024 prescriptions found in outpatient pharmacies of each hospital selected through systematic random sampling over one year period from July to September 2013.

Results: In this work, the mean number of drugs per prescription was $2.1 \pm 0.5$. Generic, antibiotics and injection prescribing were found to be $79.2 \%, 54.7 \%$ and $28.3 \%$ respectively. Less than half $(45.3 \%)$ of these sampled prescriptions had diagnosis for which drugs are indicated. Whereas drugs prescribed from essential drug list/ formulary of the country constituted $83.0 \%$ which is far less than the ideal limit.

Conclusion: The findings in this study are similar to what had been reported by most of the previous studies. Generally, all the prescribing indicators studied are out of the ranges recommended by WHO implying that there is deep rooted irrational prescribing practice in hospitals of Ethiopia. Therefore, urgent and well organized interventions should be implemented by federal ministry of health and drug regulatory bodies found at different levels in order to foster rational drug use in the country.
\end{abstract}

Keywords: Prescribing indicators; WHO; Rational drug use; Antibiotics; Injections

\section{Introduction}

Indicators of prescribing practice measure the performance of health care providers in several key dimensions related appropriate use of drugs. The indicators are based on the practice observed in samples of clinical encounters taking place at outpatient health facilities for the treatment of acute or chronic illnesses. World Health Organization developed a core prescribing indicators to measure the degree of polypharmacy, the tendency to prescribe drugs by generic name and the overall level of use of antibiotics and injections. The degree to which the prescribing practice conformed to the essential drug list, formulary or standard treatment guideline were also measured by searching for the number of drugs prescribed from essential drug list available [1]. Prescribers can only treat patients in a rational way if they have access to an essential drugs list and essential drugs are available on a regular basis [2].

In the absence of such facility related factors, the risk of irrational prescribing could raise several folds. Irrational use of drugs is a major health problem of present day medical practice. This in turn leads to different consequences including but not limited to ineffective treatment, unnecessary prescription of drugs particularly antimicrobials and injections, development of resistance to antibiotics, adverse effects and economic burden on both patients and society. It has been estimated that $50 \%$ or more medicine expenditure is being wasted through irrational prescribing, dispensing and patient use of medicine [3].

Irrational prescriptions and use of drugs has for long been known to be a feature of health care settings of developing countries, and is characterized by polypharmacy, excessive use of antibiotics and injections and use of drugs of doubtful efficacy [4]. This obviously compromises the provision of good quality health care with the available resources allocated to drug supply in these countries.

Several studies which have been conducted on prescribing practices of physicians at outpatient settings globally [2-5] as well as in Ethiopia [6-9] identified high degree of irrational prescribing especially with regard to antibiotics and injection overuse. However, periodic assessment of the prescribing practices in a health facility is still necessary to identify specific drug use problems, sensitize practitioners on rational drug prescription and provide policy makers with relevant information that could be useful in review of drug procurement policies and implementation of policies on drug prescribing practices in the affected institutions and regions.

This retrospective basic cross-sectional survey was therefore meant to analysis the prescribing practice of clinicians using World Health Organization (WHO) prescribing indicators at four selected public hospitals found in west Ethiopia.

${ }^{*}$ Corresponding author: Jimma Likisa Lenjisa , Department of Pharmacy, College Of Medicine and Health Sciences, Clinical Pharmacy Unit, Ambo University, Ethiopia, Tel: +2519175585; E-mail: jimmapharm@gmail.com

Received April 10, 2014; Accepted July 17, 2014; Published August 07, 2014

Citation: Lenjisa JL, Fereja TH (2014) A Retrospective Analysis of Prescribing Practices through WHO Prescribing Indicators at Four Selected Hospitals of West Ethiopia. J Bioanal Biomed 6: 029-032. doi:10.4172/1948-593X.1000105

Copyright: (c) 2014 Lenjisa JL, et al. This is an open-access article distributed under the terms of the Creative Commons Attribution License, which permits unrestricted use, distribution, and reproduction in any medium, provided the original author and source are credited. 


\section{Methods and materials}

\section{Study area and period}

This study was conducted from January 2013 to September 2013 at four selected public general hospitals located in western part of Ethiopia. These hospitals are found at different distances ranging from $126 \mathrm{kms}$ to about $500 \mathrm{kms}$ from the capital city; Addis Ababa. The four facilities were selected systematically out of the eight public hospitals found in this region for the purpose of this study.

\section{Study design}

This study was a descriptive basic cross-sectional survey as per WHO drug use indicators study. A retrospective analysis of 2024 prescriptions found in outpatient pharmacies of each hospital was done by well-trained pharmacists after selecting the prescriptions through systematic random sampling over one year period from the time of data collection. Prescription we used the standard data collection tool developed by World Health Organization for assessing prescribing indicators at health facilities. These include average number of drugs per prescription, percentage of drugs prescribed by generic name, percentage of prescriptions containing antibiotics, percentage of prescriptions containing injectable drugs, and percentage of drugs prescribed from the latest edition of national Essential Drug Lists (EDL) or formulary. Beside these core indicators, we have also looked at the percentage of prescription with working diagnosis which does have an important implication for pharmacists to determine whether or not the prescribed medication is appropriate for the indication under treatment.

\section{Data analysis}

Data analysis was carried out using computer software called Statistical Package for Social Sciences (SPSS) version 20.0. After data was entered and cleaned carefully, different prescribing indicators were computed using the following formula adopted from the WHO's manual for prescribing indicators assessment [1].

1. Average number of drugs per encounter=Total number of drugs prescribed/Total number of encounters sampled.

2. Percentage of drugs prescribed by generic name $=($ Number of drugs prescribed by generic name/Total number of drugs prescribed) $\mathrm{x} 100$

3. Percentage of encounters with an antibiotic prescribed $=$ (Number of patient encounters with an antibiotic/ Total number of encounters sampled) x100

4. Percentage of encounters with an injection prescribed $=$ (Number of patient encounters with an injection prescribed/Total number of encounters sampled) $\mathrm{x} 100$

5. Percentage of drugs prescribed from essential drugs list $=$ (Number of drugs prescribed from essential drugs list/
Total number of prescribed drugs) $x 100$.

In addition to these, percentage of prescriptions with diagnosis was calculated as number of prescriptions with diagnosis over the total number of sampled prescriptions.

After enumeration, the result of continuous variable in this case average number of drugs per prescription was placed as mean \pm std. error of mean while others were placed as number (\%). The results were presented using tables and figures.

\section{Results}

In this study we analyzed 2024 prescriptions found at outpatient pharmacies of each sampled hospital retrospectively to investigate the current drug prescribing practice at these institutions in west Ethiopia and hence determine the trends in rational use of drugs in the country. As table 1 show, overall, $79.2 \%$ of drugs were prescribed in generic form with a range of $70.7 \%$ to $91.1 \%$ in the sampled hospitals. Drugs prescribed from the facility's formulary constituted a total of $83.0 \%$ ranging from 73.5 to $100 \%$ with respective to hospitals. Of the total sampled prescriptions, only less than half of them (0-98.4\%) bear diagnosis for which drugs are indicated. On average, each prescription carries $2.1 \pm 0.3$ drugs with a range of 1.9 to 2.3 when looked at sampled hospitals in the study.

Likewise, looking at the antibiotics prescribing tendency of these hospitals, it was discovered that antibiotics prescribing is as high as $54.7 \%$ overall and ranges from $45.9 \%$ to $69.2 \%$ with respect to each sampled hospitals. Moreover, prescriptions having injectable are also significant in which a total of $28.3 \%$ of them found to have so with a minimum of $10.2 \%$ and maximum of $36.1 \%$ at respective selected facilities. The following figure 1 and 2 below show these facts respectively.

\section{Discussion}

This paper presented the findings on the prescribing habits of public hospitals in west Ethiopia assessed through WHO core prescribing indicators with additional one complementary indicator (percent of prescription containing diagnosis). The results were discussed as follow comparing with ideal values recommended by WHO for each core indicator as well as comparing with previous similar works done locally and globally.

Poly pharmacy if present is one of the essential indicators of potential drug-drug interactions, risk of fatal combined or synergistic medication side effects, medication non-adherence and hence poor treatment outcomes that even can lead to death. As a measure of poly pharmacy, the average number of drugs per encounter as a whole was determined to be 2.1 in this study showing the presence of overprescribing in hospitals of west Ethiopia as per the WHO recommend limit which is less than 2 [1].

Even if this finding is within the range of results reported from

\begin{tabular}{|c|c|c|c|c|c|}
\hline Indicators & $\underset{(n=500)}{A H}$ & $\begin{array}{c}\text { GH } \\
(n=500)\end{array}$ & $\underset{(n=512)}{\mathrm{NH}}$ & $\underset{(n=512)}{\text { GIH }}$ & $\begin{array}{c}\text { Total } \\
(n=2024)\end{array}$ \\
\hline N (\%) Generic drugs & $743(72.8)$ & $1040(91.1)$ & $771(81.0)$ & $752(70.7)$ & $3306(79.2)$ \\
\hline N (\%) Drug from EDL/Formulary & $776(76.1)$ & $1142(100)$ & $768(80.7)$ & $781(73.5)$ & $3467(83.0)$ \\
\hline N (\%) Prescription with Diagnosis & $5(1)$ & $408(81.6)$ & $0(0)$ & $504(98.4)$ & $917(45.3)$ \\
\hline Average number of Drug per encounter & $2.0 \pm 0.2$ & $2.3 \pm 0.5$ & $1.9 \pm 0.7$ & $2.1 \pm 0.1$ & $2.1 \pm 0.3$ \\
\hline
\end{tabular}

n; number of prescriptions sampled, AH: Ambo Hospital, GH: Gedo Hospital, NH: Nekemte Hospital, GIH: Gimbi Hospital, EDL: Essential Drug List, WHO: World Health Organization

Table 1: Some WHO Prescribing Indicators of Drug Use at Four Selected Hospitals in West Ethiopia, Jan-Sept 2013. 


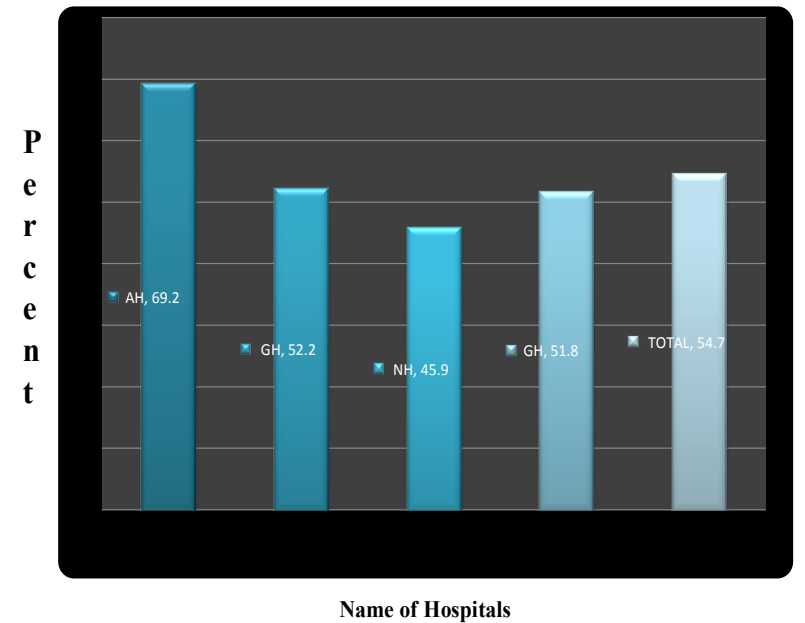

Figure 1: Percentages of prescriptions bearing antibiotics versus selected hospitals, west Ethiopia, Jan-Dec 2013

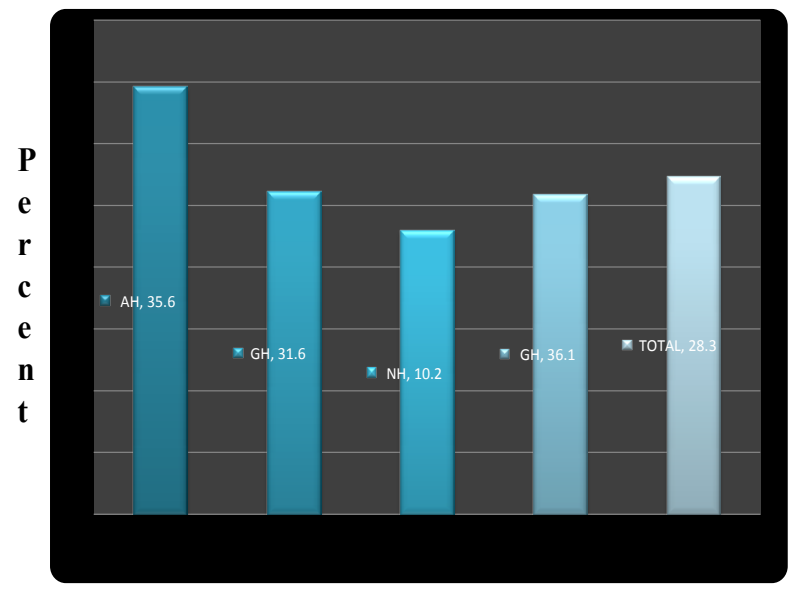

Name of Hospitals

Figure 2: Percentage of Prescriptions having injection versus selected hospitals, west Ethiopia, Jan-Sept 2013.

other parts of the country [8-10], it seems the world wide problem as studies from different corner of the globe also reported similar but higher figures ranging from 2.2 to 3.81 [2-7]. In general, such findings call for urgent and well organized interventions by pharmaceutical policy makers of many countries in the world to tackle this bothersome problem of irrational prescribing of drugs at health facilities.

Moreover, from day to day observations as well as from studies $[3,10]$, in countries like Ethiopia, the problem of overprescribing of drugs in private health facilities is expected to be worse than public health institutions. This clearly shows the need for more confirmatory studies to know the magnitude of the problem and hence interventions to further ensure rational use of medicines in such countries; the key to treatment.

World health organization recommends prescribing in generic name in a facility to be $100 \%$ as increasing generic prescribing would promote rational use of drugs avoids confusion during dispensing and reduce the cost of securing brand drugs [1]. The $79.2 \%$ prescription with generic name in this study is less than what are reported from south $(98.7 \%)$ and south west $(92 \%)$ Ethiopia $[8,10]$.
However, there are studies which showed that generic prescribing make up less than $50 \%$ most of which are conducted in private health facilities [4,11-15] which reported generic prescribing of $0 \%$ to 42.7\%. As evidences show, generic prescribing measures the quality of prescribing in a given health facility and the cost of prescribed medications can determine the compliance level of the patients [16-18].

In addition, there are situations where generic prescribing is mandatory like drugs with narrow therapeutics window or when formulations have different bioavailability having varying dosing frequencies [19]. Hence, prescribers working in different health facilities of west Ethiopia as well as in countries where such problems are deep rooted should increase their level of prescribing generic medicines in order to fully utilize its advantages in patient care.

Essential drug list/formulary of a given country is developed to promote rational use of medicines and also help to practice the most economic prescribing in health facilities. Nevertheless, prescribing of drugs from essential drug list or formulary of Ethiopia is significantly lower in our study $(83.0 \%)$ compared to the one recommended by WHO (100\%) and other studies in Ethiopia which were $96.6 \%$ and $97 \%$ in south [8] and south west [10] Ethiopia respectively. Hence, the habit of prescribers not to rely on this document in different countries and also in our study should be addressed critically through strong supervision by the responsible stakeholders, in this case, health care policy makers.

Having hypothesized that the presence of diagnosis on in prescription beside the other core prescribing indicators would help the pharmacists to identify some sort of inappropriate indications for the drug under consideration, we did determine percentage of prescriptions with diagnosis. It was found to be 45.3 (0-98.4\%) overall. Even though this figure is higher compared to the findings of studies $[12,16]$ in west Bengal (39.2\%) and in Pakistan (43.3-79.0\%), it would be better if writing diagnosis on prescription papers is a custom for prescribers in general even if not mandatory.

As much as possible antibiotic prescribing in health facilities should be low and if possible based on susceptibility test results against the responsible microorganisms. This is mainly to combat the emergence of multidrug resistant organisms which are more difficult to treat. It is for such reason that WHO recommends antibiotic prescribing should be as low as less than $30 \%$.

According to this standard reference the finding of the present study showed that there is overprescribing of antibiotics (54.7\%) at all hospitals. Nevertheless, it is better than what had been reported (58-65\%) in the country before $[8-10,20]$. In general, such irrational prescribing of antibiotics is very common in other African countries $[4,7,20,21]$ too that need very stringent control by drug regulatory bodies of respective country to halt the alarmingly increasing antimicrobial resistance in developing countries. Moreover, drug use evaluation researches are mandatory in this region to find out whether this vulnerable class of drugs is being prescribed rationally or not.

Injections are very expensive compared to other dosage forms and require training for administration to avoid risk associated with it. Furthermore, the use of injections can increase the risk of transmission of serious infections like hepatitis, HIV/AIDS and many more if there is no hygienic practice. To reduce these evil sides of injections, WHO encourages the prescribing of injectable to be as low as less than $10 \%$. However, except very few studies $[2,4,13]$ reporting range of injection prescribing from $0.17 \%$ to $4 \%$, overprescribing of injections (up to $46 \%$ ) in most studies $[5,7,14,22,23]$ is common including our 
study. Similarly, injection prescribing is also common in other parts of Ethiopia where it ranges from $23 \%$ to $38.1 \%[8,10,20]$ showing the importance of prescription auditing by regulatory bodies there by taking appropriate steps to tackle the problems.

\section{Limitation of the Study}

Prescribing indicators based drug utilization study in health facilities is not an exhaustive tool to identify all problems related to prescribing and rationality of drug use as they don't exactly explain why drugs are prescribed.

\section{Conclusion}

In general, the findings of this study are in line what had been reported locally and globally from previous similar works. Average number of drugs per encounter, generic prescribing, and the use of EDL/formulary to prescribe drugs in this study is totally out of the recommended values and hence inappropriate. Moreover, overprescribing of antibiotics and injections is also the main problems identified in this study. Based on these findings, therefore, we put forward the following recommendations for policy makers to ensure rational use of drugs in the country. Firstly, there should be functional drug and therapeutic committee in all hospitals of Ethiopia which are like toothless dogs and only paper value today though they are believed to be effective weapons to fight irrational use of medicines. Secondly, there should be countrywide continuous sensitization trainings and workshops for prescribers and pharmacists working in hospitals of Ethiopia on rational use of medicines especially antibiotics or else we are going to lose them secondary to resistance. Last but not least, Ethiopia should fully utilize the knowledge of clinical pharmacists in outpatient departments of all health facilities in order to help prescribers in drug selection for patients thereby promoting the rational and economic use of medicines in the country.

\section{Acknowledgement}

This work was supported by Ambo University. Our heartfelt gratitude goes to pharmacy department staff of all study hospitals for their cooperation during data collection without which this work is not possible.

\section{Declarations}

\section{Funding}

The study was funded by Ambo University being one of the outstanding staff research projects reviewed in 2013 as per the university's research and technology transfer office. Ethical approval was obtained from the same office with reference number RTT/120/013.

\section{References}

1. WHO (1993) How to investigate drug use in health facilities: selected drug use indicators. 1-92.

2. Sunil K, Punam S, Madhuri K (2005) Patterns of Prescription and Drug Dispensing. Indian J Pediatr 72: 117-121.

3. Binu M, Sabbu R, Surendra K, Hiremath D (2013) Assessment Of Drug Prescribing Practices Using Who Prescribing Indicators In A Private Tertiary Care Teaching Hospital. IRJIPS 1: 26-31.

4. Igbiks T, Joseph O (2012) Drug Prescription Pattern in a Nigerian Tertiary Hospital. TJPR 11: 146-152.

5. Rahman Z, Rumana N, Mahmuda B (2009) Evaluation of prescribing pattern of the private practitioners by the undergraduate medical students. Bangladesh $\mathrm{J}$ Pharmacol 4: 73-75.
6. Vania DS, Sandra MO, Oliveira N (2004) Prescription and patient care indicators in healthcare services. Rev Saude Publica 38: 1-7.

7. Enwere OO, Salako BL (2007) Drug prescribing pattern at the medical outpatient clinic of a tertiary hospital in southwestern Nigeria. Pharmacoepidemiol Drug Saf 16: 1244-1249.

8. Anteneh AD (2013) Assessment of drug use pattern using WHO prescribing indicators at Hawassa University teaching and referral hospital, south Ethiopia: a cross-sectional study. Desalegn BMC Health Services Research 13: 170.

9. Mulugeta TA, Nasir TW, Raju NJ (2011) Assessment of Patterns of Drug use by using World Health Organization's Prescribing, Patient Care and Health facility indicators in Selected Health Facilities in Southwest Ethiopia . japs 01: 62-66.

10. Bayew T, Wubshet H, Zeryawkal E (2013) Do private health institutions prescribe drugs more irrationally than the publics? A yearlong comparative evaluation of prescribing indicators, wolkite town, south west Ethiopia. Ind J Pharm Prac 6: 16-20.

11. Igbiks T (2011) Prescription pattern of clinicians in private health facilities in Kano, Northwestern Nigeria. APJTD 235-238.

12. Hafeez A, Kiani AG, Din S, Butt K, Mirza Z, et al. (2004) Prescription and Dispensing Practices in Public Sector Health Facilities in Pakistan: Survey Report. JPMA 54: 187-191.

13. Bhavesh kL, Hiray RS, Ghongane BB (2012) Drug prescription pattern of outpatients in a tertiary care Teaching hospital in Maharashtra. Int J Pharm Bio Sci 3:225 - 229

14. Bashrahil KA (2010) Indicators of rational drug use and health services in Hadramout, Yemen. EMHJ 16: 151-155

15. Pavani V, Mihir VP, Shravani K, Prabhakar RV (2011) Study of prescribing pattern of evaluation of rational drug therapy in Warangal. Indian J Pharm Pract 4: 77-79.

16. Koley M, Saha S, Arya JS, Choubey G, Ghosh S, et al. (2013) A study on drug utilization and prescription habits of physicians in a government homeopathic hospital in West Bengal, India. J Integr Med 11: 305-313.

17. Alyamani NA, Hopf Y, Williams DJ (2009) Prescription quality in acute medical ward. Pharmacoepidemiol Drug Saf 18: 1158-1165.

18. Maiga MD, Diawara A (2006) study on the availability and cost of medicines in the private sectors in Mali. Med Trop 66: 565-568.

19. Information service divisions (2008) Generic prescribing. Edinburgh, Scotland

20. Assessment of the Pharmaceutical Sector in Ethiopia (2003). Addis Ababa.

21. Bannenberg WJ, Forshaw CJ, Fresle D, Salami AO, Wahab HA (1991) Evaluation of the Nile province essential drug project. WHO: Geneva.

22. Das A, Divan V, Massele A, Chowdhary A, Kafle KK, et al. (1993) How to investigate drug use in health facilities. WHO: 1-74.

23. Lifang D, Hong $Y$, Duolao $W$ (2011) Drug prescribing indicators in village health clinics across 10 provinces of Western China. Family Practice 28: 63-67.

24. Nsimba SE (2006) Assessing prescribing and patient care Indicators for children under five years old with malaria and other disease conditions in Public primary health care facilities. Southeast Asian J Trop Med Public Health 37: 206-214. 